\title{
THE ROLE OF CERTAIN BRAUER AND RADO RESULTS IN THE NONNEGATIVE INVERSE SPECTRAL PROBLEMS*
}

\author{
ANA I. JULIO ${ }^{\dagger}$ AND RICARDO L. SOTO ${ }^{\dagger}$
}

\begin{abstract}
It is said that a list $\Lambda=\left\{\lambda_{1}, \ldots, \lambda_{n}\right\}$ of complex numbers is realizable, if it is the spectrum of a nonnegative matrix $A$. It is said that $\Lambda$ is universally realizable if it is realizable for each possible Jordan canonical form allowed by $\Lambda$. This work does not contain new results. As its title says, its goal is to show and emphasize the relevance and importance of certain results, by Brauer and Rado, in the study of nonnegative inverse spectral problems. It is shown that virtually all known results, which give sufficient conditions for $\Lambda$ to be realizable or universally realizable, can be obtained from results by Brauer and Rado. Moreover, from these results, a realizing matrix may always be constructed.
\end{abstract}

Key words. Nonnegative realizability of spectra, Nonnegative inverse eigenvalue problem, Universal realizability of spectra, Brauer's result, Rado's result.

AMS subject classifications. 15A18, 15A51.

1. Introduction. The nonnegative inverse eigenvalue problem is the problem of characterizing all possible spectra of entrywise nonnegative matrices. If there exists a nonnegative matrix $A$ with spectrum $\Lambda=\left\{\lambda_{1}, \lambda_{2}, \ldots, \lambda_{n}\right\}$, we say that $\Lambda$ is realizable and that $A$ is the realizing matrix. The first element of a realizable list $\Lambda=\left\{\lambda_{1}, \lambda_{2}, \ldots, \lambda_{n}\right\}$, i.e., $\lambda_{1}$, is the Perron eigenvalue of the realizing matrix. In the general case, when $\Lambda$ is a list of complex numbers, the problem was solved, for $n=3$, by Loewy and London [20], and for $n=4$, by Meehan [24], and, independently, by Torre-Mayo et al. [50]. The case $n=5$ was solved for realizing matrices of trace zero, by Laffey and Meehan [19]. The NIEP remains unsolved for $n \geq 5$. When $\Lambda$ is a list of real numbers, the NIEP is called the real nonnegative inverse eigenvalue problem $($ RNIEP), and a number of sufficient conditions for the existence of a solution are known (see [34, 37, 43] and the references therein). If the realizing matrix is required to be symmetric we have the symmetric nonnegative inverse eigenvalue problem (SNIEP), which has been solved for $n=5$ with realizing matrices of trace zero by Spector [48]. For $n \leq 4$, the RNIEP and the SNIEP are equivalent, while for $n \geq 5$, they are different [13]. A number of sufficient conditions for the existence of a symmetric nonnegative matrix with prescribed spectrum have also been obtained (see [38, 39, 43] and the references therein).

We say that a list of complex numbers $\Lambda=\left\{\lambda_{1}, \lambda_{2}, \ldots, \lambda_{n}\right\}$ is universally realizable $(U R)$, if $\Lambda$ is realizable for each possible Jordan canonical form $(J C F)$ allowed by $\Lambda$. The problem of the universal realizability of spectra is called the universal realizability problem $(U R P)$.

A set $\mathcal{K}$, of conditions, is said to be a realizability criterion if any list $\Lambda$ of complex numbers, satisfying the conditions $\mathcal{K}$, is realizable. A real matrix $A=\left(a_{i j}\right)_{i, j=1}^{n}$ is said to have constant row sums if all its rows sum up the same constant, say $\alpha$, that is, $\sum_{j=1}^{n} a_{i j}=\alpha, i=1, \ldots, n$. The set of all real matrices with constant row sums equal to $\alpha$, will be denoted by $\mathcal{C} \mathcal{S}_{\alpha}$. It is clear that any matrix in $\mathcal{C S}_{\alpha}$ has an eigenvector $\mathbf{e}=(1, \ldots, 1)^{T}$ corresponding to the eigenvalue $\alpha$. We denote by $\mathbf{e}_{i}=(0, \ldots, 1, \ldots, 0)^{T}$ the $i$-th column

* Received by the editors on October 22, 2019. Accepted for publication on June 6, 2020. Handling Editor: Raphael Loewy. Corresponding Author: Ana I. Julio.

†Departamento de Matemáticas, Universidad Católica del Norte, Antofagasta, Casilla 1280, Chile (ajulio@ucn.cl, rsoto@ucn.cl). Work supported by Conicyt-PAI 79160002, 2016, Chile, Fondecyt 1170313. 
Electronic Journal of Linear Algebra, ISSN 1081-3810

A publication of the International Linear Algebra Society

Volume 36, pp. 484-502, July 2020.

of the identity matrix of the appropriate size. The importance of real matrices with constant row sums, is due to the fact that the problem of finding a nonnegative matrix with spectrum $\Lambda=\left\{\lambda_{1}, \lambda_{2}, \ldots, \lambda_{n}\right\}$, is equivalent to the problem of finding a nonnegative matrix in $\mathcal{C S}_{\lambda_{1}}$ (see [12]).

The purpose of this work is to examine the NIEP and the URP, from Brauer and Rado results point of view [3, 28]. In particular, we show that, virtually all known results, which give realizability criteria for the NIEP and the URP to have a solution, may be obtained by applying certain results by Brauer or Rado, which we identify in Section 2. Moreover, the proofs from Brauer and Rado results are constructive, in the sense that they allow us to construct a realizing matrix.

Brauer's Theorem shows how to modify one single eigenvalue of a matrix, via a rank-one perturbation, without changing any of the remaining eigenvalues. This, together with the properties of real matrices with constant row sums, are the basic ingredients of the technique that has been used in most cases, and it suggests that Brauer's Theorem can be a very useful tool to deal with the NIEP and the URP. This approach goes back to Perfect, who first used it in [27] to obtain sufficient conditions for the NIEP to have a solution. It was somehow abandoned for many years, until in [34], the author rediscovered it to obtain sufficient conditions for the realizability of partitioned real spectra, with the partition allowing some of its pieces to be nonrealizable.

Rado's Theorem, is an extension of Brauer's Theorem, which shows how to modify $r$ eigenvalues of a matrix of order $n, r<n$, via a rank- $r$ perturbation, without changing any of the remaining $(n-r)$ eigenvalues. Rado's Theorem was introduced and applied by Perfect [28], to derive an important realizability criterion for the RNIEP. Surprisingly, this result was also ignored in the literature about the problem, until in [37], the authors rescue it and extend it to a new realizability criterion. Theorem 2.6 in Section 2, is a symmetric version of Rado's Theorem and it was introduced in [39]. There, by the use of Theorem 2.6, the authors give a criterion for the symmetric realizability of a list $\Lambda=\left\{\lambda_{1}, \ldots, \lambda_{n}\right\}$ of real numbers. This criterion, by its own definition, trivially contains any other sufficient condition for the SNIEP to have a solution.

There are a number of known realizability criteria, which have been obtained from the results by Brauer and/or Rado. Obviously they are not included in this paper (see [2, 27, 28, 34, 37, 43] for the RNIEP, $[36,38,39,43]$ for the SNIEP, [2, 31, 41, 42] for the complex case, and $[7,8,16,40,44,45,46,47]$ for the $U R P)$.

The paper is organized as follows: In Section 2, we introduce the Theorems by Brauer and Rado, mentioned above. In Section 3, from Brauer's Theorem point of view, we prove some of Guo's results [11]. In Section 4, we give alternative proofs of realizability criteria of Suleimanova [49], Salzmann [32], Kellogg [18], Ciarlet [6], Borobia [1], and Šmigoc [33], by applying Brauer's Theorem. In Section 5, we consider results related to SNIEP. In particular, from the symmetric version of Rado's Theorem, we prove two results by Fiedler [10]. In Section 6, we consider results associated with spectra of complex numbers, and we give, from Rado's Theorem point of view, a proof of a result by Šmigoc [33]. Finally, In Section 7, we examine the universal realizability problem $(U R P)$, and give an alternative proof of one of Minc's result in [26].

2. Brauer and Rado theorems. The Brauer and Rado results have proven to be important for the study of the NIEP and the URP. They have been applied with success to generate sufficient conditions for the NIEP and the URP to have a solution. These two theorems will be the sole results used, throughout this paper, to give alternative proofs of distinct realizability criteria compared in the maps constructed in $[22,23]$. We show that virtually all known realizability criteria for the NIEP and the URP can be obtained 
by applying Brauer's Theorem or Rado's Theorem.

Theorem 2.1. (Brauer, [3]) Let $A$ be an $n \times n$ arbitrary matrix with eigenvalues $\lambda_{1}, \lambda_{2}, \ldots, \lambda_{n}$. Let $\mathbf{v}=\left(v_{1}, v_{2}, \ldots, v_{n}\right)^{T}$ be an eigenvector of $A$ associated with the eigenvalue $\lambda_{k}$ and let $\mathbf{q}$ be any $n$-dimensional vector. Then the matrix $A+\mathbf{v q}^{T}$ has eigenvalues $\lambda_{1}, \lambda_{2}, \ldots, \lambda_{k-1}, \lambda_{k}+\mathbf{v}^{T} \mathbf{q}, \lambda_{k+1}, \ldots, \lambda_{n}$.

Another proof, simpler than the one given in [3], can be found in [30]. An immediate consequence of Brauer's Theorem is:

Corollary 2.2. If $\Lambda=\left\{\lambda_{1}, \ldots, \lambda_{n}\right\}$ is realizable, then $\Lambda_{\epsilon}=\left\{\lambda_{1}+\epsilon, \lambda_{2}, \ldots, \lambda_{n}\right\}, \epsilon \geq 0$, is also realizable.

Proof. There exists a nonnegative matrix $A$ with spectrum $\Lambda$, which can be taken as $A \in \mathcal{C S}_{\lambda_{1}}$. Then, the matrix $A_{\epsilon}=A+\epsilon \mathbf{e e}_{1}^{T}$ is nonnegative and, from Theorem 2.1, it has spectrum $\Lambda_{\epsilon}$.

In [2], the authors introduce the concept of Brauer's negativity, a quantity reflecting, in a certain way, how far $\Lambda$ is from being realized as the spectrum of a nonnegative matrix. This negativity can be diminished by joining the list with a realizable list, at best until the negativity is fully compensated and the joint list becomes realizable. Then we have:

Definition 2.3. Given a list $\Lambda=\left\{\lambda_{1}, \lambda_{2}, \ldots, \lambda_{n}\right\}$, the Brauer's negativity of $\Lambda$ is

$$
\mathcal{N}(\Lambda) \equiv \min \left\{\delta \geq 0:\left\{\lambda_{1}+\delta, \lambda_{2}, \ldots, \lambda_{n}\right\} \text { is realizable }\right\}
$$

Note that a list $\Lambda$ is realizable if and only if $\mathcal{N}(\Lambda)=0$.

The following result is an extension of Theorem 2.1, and shows how to modify $r$ eigenvalues of a matrix of order $n, r<n$, via a rank $-r$ perturbation, without changing any of the $n-r$ remaining eigenvalues. This result was introduced by Perfect in [28]. There, she points out that the result and its proof are due to R. Rado.

Theorem 2.4. (Rado, [28]) Let $A$ be an $n \times n$ arbitrary matrix with eigenvalues $\lambda_{1}, \lambda_{2}, \ldots, \lambda_{n}$. Let $X=\left[\mathbf{x}_{1}\left|\mathbf{x}_{2}\right| \cdots \mid \mathbf{x}_{r}\right]$ be such that $\operatorname{rank}(X)=r$ and $A \mathbf{x}_{i}=\lambda_{i} \mathbf{x}_{i}, i=1,2, \ldots, r, r \leq n$. Let $C$ be an $r \times n$ arbitrary matrix. Then the matrix $A+X C$ has eigenvalues $\mu_{1}, \ldots, \mu_{r}, \lambda_{r+1}, \ldots, \lambda_{n}$, where $\mu_{1}, \ldots, \mu_{r}$ are eigenvalues of the matrix $\Omega+C X$ with $\Omega=\operatorname{diag}\left\{\lambda_{1}, \ldots, \lambda_{r}\right\}$.

Observe that for $r=1$, Rado's Theorem is Brauer's Theorem. The following example shows the importance of Theorem 2.4. In [37], the authors give a sufficient condition, which allow us, not only to decide on the realizability of the list $\Lambda=\{6,3,3,-5,-5\}$, but also to construct a realizing matrix. Although to some degree tedious, it is easy to check that no other realizability criterion, distinct from the one given in [37], is satisfied by $\Lambda$.

Example 2.5. [37] Consider $\Lambda=\{6,3,3,-5,-5\}$. We define the partition $\Lambda_{0}=\{6,3,3\}, \Lambda_{1}=\Lambda_{2}=$ $\{-5\}, \Lambda_{3}=\emptyset$, with the associated realizable lists $\Gamma_{1}=\Gamma_{2}=\{5,-5\}, \Gamma_{3}=\{2\}$. The matrices

$$
A_{1}=A_{2}=\left[\begin{array}{ll}
0 & 5 \\
5 & 0
\end{array}\right], \quad A_{3}=[2]
$$


Electronic Journal of Linear Algebra, ISSN 1081-3810

A publication of the International Linear Algebra Society

Volume 36, pp. 484-502, July 2020.

realize the lists $\Gamma_{1}=\Gamma_{2}$ and $\Gamma_{3}$, respectively, and the matrix

$$
A=\left[\begin{array}{ccc}
A_{1} & 0 & 0 \\
0 & A_{2} & 0 \\
0 & 0 & A_{3}
\end{array}\right]
$$

has the spectrum $\Gamma_{1} \cup \Gamma_{2} \cup \Gamma_{3}$. To apply the Theorem 2.4 we need to compute a $3 \times 3$ nonnegative matrix with spectrum $\Lambda_{0}$ and diagonal entries 5,5,2. From a Perfect's result [28, Theorem 4 ] it is

$$
B=\left[\begin{array}{lll}
5 & 0 & 1 \\
1 & 5 & 0 \\
0 & 4 & 2
\end{array}\right] .
$$

Then, for

$$
X=\left[\begin{array}{lll}
1 & 0 & 0 \\
1 & 0 & 0 \\
0 & 1 & 0 \\
0 & 1 & 0 \\
0 & 0 & 1
\end{array}\right], \quad C=\left[\begin{array}{lllll}
0 & 0 & 0 & 0 & 1 \\
1 & 0 & 0 & 0 & 0 \\
0 & 0 & 4 & 0 & 0
\end{array}\right]
$$

where the columns of $X$ are eigenvectors of $A$ and $C$ is obtained from $B$ in a certain appropriate way (see $[28,37])$, we have that

$$
M=A+X C=\left[\begin{array}{lllll}
0 & 5 & 0 & 0 & 1 \\
5 & 0 & 0 & 0 & 1 \\
1 & 0 & 0 & 5 & 0 \\
1 & 0 & 5 & 0 & 0 \\
0 & 0 & 4 & 0 & 2
\end{array}\right]
$$

is nonnegative with spectrum $\Lambda$.

It was proven by Johnson, Marijuán and Pisonero [14, Theorem 1] and by Loewy and Spector [21, Theorem 5.1], that $\Lambda=\{6,3,3,-5,-5\}$ is not symmetrically realizable.

In [39], the authors prove the following symmetric version of Theorem 2.4.

TheOREm 2.6. ([39]) Let $A$ be an $n \times n$ symmetric matrix with eigenvalues $\lambda_{1}, \lambda_{2}, \ldots, \lambda_{n}$. Let $\left\{\mathbf{x}_{1}, \mathbf{x}_{2}\right.$, $\left.\ldots, \mathbf{x}_{r}\right\}$ be an orthonormal set of eigenvectors of $A$ such that $A X=X \Omega$, where $X=\left[\mathbf{x}_{1}\left|\mathbf{x}_{2}\right| \cdots \mid \mathbf{x}_{r}\right]$ and $\Omega=\operatorname{diag}\left\{\lambda_{1}, \lambda_{2}, \ldots, \lambda_{r}\right\}$. Let $C$ be any $r \times r$ symmetric matrix. Then the symmetric matrix $A+X C X^{T}$ has eigenvalues $\mu_{1}, \ldots, \mu_{r}, \lambda_{r+1}, \ldots, \lambda_{n}$ where $\mu_{1}, \ldots, \mu_{r}$ are eigenvalues of the matrix $\Omega+C$.

3. On Guo results. An important result by Guo [11, Theorem 3.1], which we prove using Theorem 2.4, establishes that:

TheOREm 3.1. Let $\Lambda=\left\{\lambda_{1}, \lambda_{2}, \ldots, \lambda_{n}\right\}, \lambda_{2} \in \mathbb{R}$, be realizable. Then, for any $\epsilon>0, \Lambda_{\epsilon}=\left\{\lambda_{1}+\epsilon\right.$, $\left.\lambda_{2} \pm \epsilon, \ldots, \lambda_{n}\right\}$ is also realizable.

Proof. First we consider the case $\Lambda_{+\epsilon}=\left\{\lambda_{1}+\epsilon, \lambda_{2}+\epsilon, \ldots, \lambda_{n}\right\}$. Let $\Lambda=\left\{\lambda_{1}, \lambda_{2}, \ldots, \lambda_{n}\right\}$ be realizable with realizing matrix $A \in \mathcal{C S}_{\lambda_{1}}$. Then $A \mathbf{e}=\lambda_{1} \mathbf{e}$. Let $A \mathbf{x}=\lambda_{2} \mathbf{x}$, with $\mathbf{x}^{T}=\left(x_{1}, x_{2}, \ldots, x_{n}\right), x_{1}=$ 
$\max \left\{x_{1}, x_{2}, \ldots, x_{n}\right\}, x_{2}=\min \left\{x_{1}, x_{2}, \ldots, x_{n}\right\}$. It is clear that $x_{1} \geq 0$ and $x_{2} \leq 0$. Let

$$
X=\left[\begin{array}{cc}
1 & x_{1} \\
1 & x_{2} \\
\vdots & \vdots \\
1 & x_{n}
\end{array}\right], \quad C=\left[\begin{array}{ccccc}
c_{11} & c_{12} & \cdots & \cdots & c_{1 n} \\
c_{21} & c_{22} & \cdots & \cdots & c_{2 n}
\end{array}\right], \quad \text { and } \quad \Omega=\left[\begin{array}{cc}
\lambda_{1} & 0 \\
0 & \lambda_{2}
\end{array}\right]
$$

with

$$
\begin{aligned}
& c_{11}=\frac{-\epsilon x_{2}}{x_{1}-x_{2}}, \quad c_{12}=\frac{\epsilon x_{1}}{x_{1}-x_{2}}, \quad c_{1 j}=0, \quad j=3, \ldots, n \\
& c_{21}=\frac{\epsilon}{x_{1}-x_{2}}, \quad c_{22}=\frac{-\epsilon}{x_{1}-x_{2}}, \quad c_{2 j}=0, \quad j=3, \ldots, n .
\end{aligned}
$$

Then

$$
X C=\left[\begin{array}{ccccc}
c_{11}+c_{21} x_{1} & c_{12}+c_{22} x_{1} & 0 & \cdots & 0 \\
c_{11}+c_{21} x_{2} & c_{12}+c_{22} x_{2} & 0 & \cdots & 0 \\
\vdots & \vdots & \vdots & \ddots & \vdots \\
c_{11}+c_{21} x_{n} & c_{12}+c_{22} x_{n} & 0 & \cdots & 0
\end{array}\right] \geq 0 \quad \text { and } \quad C X=\left[\begin{array}{cc}
\epsilon & 0 \\
0 & \epsilon
\end{array}\right]
$$

Now, from Theorem 2.4, $A+X C$ is nonnegative with spectrum $\Lambda_{+\epsilon}=\left\{\lambda_{1}+\epsilon, \lambda_{2}+\epsilon, \ldots, \lambda_{n}\right\}$. For the case $\Lambda_{-\epsilon}=\left\{\lambda_{1}+\epsilon, \lambda_{2}-\epsilon, \ldots, \lambda_{n}\right\}$, we take

$$
\begin{aligned}
& c_{11}=\frac{\epsilon x_{1}}{x_{1}-x_{2}}, \quad c_{12}=\frac{-\epsilon x_{2}}{x_{1}-x_{2}}, \quad c_{1 j}=0, \quad j=3, \ldots, n \\
& c_{21}=\frac{-\epsilon}{x_{1}-x_{2}}, \quad c_{22}=\frac{\epsilon}{x_{1}-x_{2}}, \quad c_{2 j}=0, \quad j=3, \ldots, n .
\end{aligned}
$$

and the result follows.

A weaker version of this result is:

LEMmA 3.2. Let $\Lambda_{1}=\left\{\alpha_{1}, \alpha_{2}, \ldots, \alpha_{n}\right\}$ and $\Lambda_{2}=\left\{\beta_{1}, \beta_{2}, \ldots, \beta_{m}\right\}$ be realizable lists. Then for any $\epsilon \geq \max \left\{\beta_{1}-\alpha_{1}, 0\right\}$,

$$
\Lambda=\left\{\alpha_{1}+\epsilon, \beta_{1}-\epsilon, \alpha_{2}, \ldots, \alpha_{n}, \beta_{2}, \ldots, \beta_{m}\right\} \text { is realizable. }
$$

A result from Guo [11, Theorem 2.1] states the existence of a real number $\lambda_{0}$,

$$
\max _{2 \leq j \leq n}\left|\lambda_{j}\right| \leq \lambda_{0} \leq 2 n \max _{2 \leq j \leq n}\left|\lambda_{j}\right|
$$

such that the list of complex numbers $\Lambda=\left\{\lambda_{1}, \lambda_{2}, \ldots, \lambda_{n}\right\}$ is realizable if and only if $\lambda_{1} \geq \lambda_{0}$. The Guo index $\lambda_{0}$ is the minimum $\lambda$ such that $\left\{\lambda, \lambda_{2}, \ldots, \lambda_{n}\right\}$ is realizable. The problem of finding $\lambda_{0}$ is not solved in Guo's paper. Here, we show that the upper bound in (3.1) may be reduced to $(n-1) \max _{2 \leq j \leq n}\left|\lambda_{j}\right|$, and that it is sharp.

TheOrem 3.3. Let $\Lambda^{\prime}=\left\{\lambda_{2}, \ldots, \lambda_{n}\right\} \subset \mathbb{C}$ be such that $\overline{\Lambda^{\prime}}=\Lambda^{\prime}$ with $\lambda_{2} \geq \cdots \geq \lambda_{p}$ real, $2 \leq p \leq n$. Then, the Guo upper bound in (3.1) may be reduced to

$$
\lambda_{0} \leq(n-1) \max _{2 \leq j \leq n}\left|\lambda_{j}\right|
$$


Proof. Let $m=\max _{2 \leq j \leq n}\left|\lambda_{j}\right|$ and let $\mu_{j}=\frac{\lambda_{j}}{m(n-1)}, j=2,3, \ldots, n$. Then, $\Gamma^{\prime}=\left\{\mu_{2}, \ldots, \mu_{n}\right\}$ is a list of complex numbers such that $\left|\mu_{j}\right| \leq \frac{1}{n-1}, j=2,3, \ldots, n$. Consider the initial matrix

$$
B=\left[\begin{array}{ccccccccc}
0 & 0 & 0 & \cdots & \cdots & \cdots & \cdots & \cdots & 0 \\
-\mu_{2} & \mu_{2} & \ddots & & & & & & \vdots \\
\vdots & \ddots & \ddots & \ddots & & & & & \vdots \\
-\mu_{p} & \vdots & \ddots & \mu_{p} & 0 & & & & \vdots \\
-x_{s} & y_{s} & & \ddots & x_{s} & -y_{s} & & & \vdots \\
-x_{s} & -y_{s} & & & y_{s} & x_{s} & \ddots & & \vdots \\
\vdots & \vdots & & & & \ddots & \ddots & \ddots & 0 \\
-x_{t} & y_{t} & & & & & \ddots & x_{t} & -y_{t} \\
-x_{t} & -y_{t} & \cdots & \cdots & \cdots & \cdots & 0 & y_{t} & x_{t}
\end{array}\right],
$$

where $\mu_{2}, \mu_{3}, \ldots, \mu_{p}$ are real, $x_{j}=R e \mu_{j}, y_{j}=\operatorname{Im} \mu_{j}, p+1 \leq j \leq \frac{n+p}{2}$. Then $B \in \mathcal{C S}_{0}$ has eigenvalues $0, \mu_{2}, \ldots, \mu_{p}, \mu_{p+1}, \ldots, \mu_{n}$.

If $R e \mu_{j} \leq 0, j=2,3, \ldots, n$, then all the entries in the first column of $B$ are nonnegative. Let

$$
\mathbf{q}=\left(0, \frac{1}{n-1}, \ldots, \frac{1}{n-1}\right)^{T}
$$

From Theorem 2.1, the matrix $A^{\prime}=B+\mathbf{e q}^{T}$ is nonnegative with eigenvalues $1, \mu_{2}, \ldots, \mu_{n}$ and the matrix $A=m(n-1) A^{\prime}$ is nonnegative with eigenvalues $(n-1) m, \lambda_{2}, \ldots, \lambda_{n}$.

If $R e \mu_{k}>0$ for some $k, 3 \leq k \leq n$, then all the entries in the $k$-th column of $B$ (or in the $(k-1)$-th column of $B$ if $k$ corresponds to the second column in the corresponding $2 \times 2$ complex block) are nonnegative. Let

$$
\mathbf{q}=\left(\frac{1}{n-1}, \ldots, \frac{1}{n-1}, 0, \frac{1}{n-1}, \ldots, \frac{1}{n-1}\right)^{T}
$$

with zero in the $k$-th position $\left((k-1)\right.$-th position). Then, the matrix $A^{\prime}=B+\mathbf{e q}^{T}$ is nonnegative with eigenvalues $1, \mu_{2}, \mu_{3}, \ldots, \mu_{n}$ and the matrix $A=m(n-1) A^{\prime}$ is nonnegative with eigenvalues $(n-$ 1) $m, \lambda_{2}, \lambda_{3}, \ldots, \lambda_{n}$.

If $\mu_{2}>0$ with $R e \mu_{j}<0, j=3, \ldots, n$, then we write the $-R e \mu_{j}^{\prime} s, 3 \leq j \leq n$, along the second column of $B$ and the $\pm I m \mu_{j}^{\prime} s, p+1 \leq j \leq n$, along the first column of $B$. Finally, with

$$
\mathbf{q}=\left(\frac{1}{n-1}, 0, \frac{1}{n-1}, \ldots, \frac{1}{n-1}\right)^{T}
$$

we obtain, as before, the nonnegative matrix $A=m(n-1) A^{\prime}$ with the required eigenvalues.

The inequality (3.2) is sharp. In fact, let $\lambda_{j}=-1,2 \leq j \leq n$. Then, from a result by Suleimanova [49, Theorem 4.1], the problem has a solution if and only if $\lambda_{1} \geq \lambda_{0}=(n-1)$. Thus, in the real case, Guo's 
result guarantees the existence of a nonnegative matrix $A$ with spectrum $\Lambda=\left\{\lambda_{1}, \lambda_{2}, \ldots, \lambda_{n}\right\}$ for all

$$
\lambda_{1} \geq(n-1) \max _{2 \leq j \leq n}\left|\lambda_{j}\right|
$$

If $\Lambda=\left\{\lambda_{1}, \lambda_{2}, \ldots, \lambda_{n}\right\}$, with $\sum_{i=1}^{n} \lambda_{i}=0$, is a realizable list of complex numbers, then $\lambda_{1}$ is the Guo's index.

REMARK 3.4. In [31], the authors show how to calculate the Guo's index $\lambda_{0}$, for circulant nonnegative matrices. There, they give a necessary and sufficient condition for a list $\Lambda=\left\{\lambda_{1}, \lambda_{2}, \ldots, \lambda_{n}\right\}$ of complex numbers to be the spectrum of a circulant nonnegative matrix. However, to calculate $\lambda_{0}$ becomes a prohibitive task for large $n$. Then, they prove, by the use of Theorem 2.1, a more manageable realizability criterion (see $[31])$.

4. RNIEP. In this section, we consider the real nonnegative inverse eigenvalue problem, and we show that the realizability criteria given by Suleimanova [49], Salzmann [32], Ciarlet [6], Kellogg [18], and Borobia [1], may all be obtained by applying Theorem 2.1. Perfect [27] was the first one to use Theorem 2.1 and Theorem 2.4 to derive sufficient conditions for the RNIEP to have a solution. There are a number of realizability criteria, which are not considered here because they were obtained from Theorem 2.1 or Theorem 2.4.

In this section, we also examine a result by Šmigoc [33, Theorem 10], which can be proved using Theorem 2.4. Since Theorem 2.4 is a generalization of Theorem 2.1, criteria from Theorem 2.4 give, in general, better information about the realizability of a given real list $\Lambda=\left\{\lambda_{1}, \ldots, \lambda_{n}\right\}$. All proofs of criteria generated from Theorem 2.1 or Theorem 2.4 are constructive. Then, they allow us to construct a realizing matrix.

Theorem 4.1. (Suleimanova, [49]) Let $\Lambda=\left\{\lambda_{1}, \lambda_{2}, \ldots, \lambda_{n}\right\}$ be a list of real numbers satisfying $\lambda_{1}+$ $\lambda_{2}+\cdots+\lambda_{n} \geq 0, \lambda_{k}<0, k=2,3, \ldots, n$. Then, $\Lambda$ is realizable.

Proof. It was proved in [34] by the use of Theorem 2.1.

TheOREM 4.2 (Salzmann [32]). Let $\Lambda=\left\{\lambda_{1}, \lambda_{2}, \ldots, \lambda_{n}\right\}$ with $\lambda_{1} \geq \lambda_{2} \geq \cdots \geq \lambda_{n}$ such that

$$
\sum_{k=1}^{n} \lambda_{k} \geq 0
$$

and

$$
\lambda_{k}+\lambda_{n-k+1} \leq \frac{2}{n} \sum_{k=1}^{n} \lambda_{k}, \quad k=2,3, \ldots,\left[\frac{n+1}{2}\right]
$$

Then, $\Lambda$ is realizable.

Proof. We only need to prove the assertion for $\Lambda$ satisfying $\sum_{k=1}^{n} \lambda_{k}=0$. First, let $n$ be even and suppose conditions (4.4) and (4.5) are satisfied with $\sum_{k=1}^{n} \lambda_{k}=0$. Then $\lambda_{k}+\lambda_{n-k+1} \leq 0$ for $k=2,3, \ldots, \frac{n}{2}$. Now, we apply [34, Theorem 11], which is a criterion obtained by applying Theorem 2.1. Let

$$
B=\left[\begin{array}{cccc}
B_{11} & 0 & \ddots & 0 \\
B_{21} & B_{22} & \ddots & \ddots \\
\ddots & \ddots & \ddots & 0 \\
B_{\frac{n}{2} 1} & \ddots & 0 & B_{\frac{n}{2} \frac{n}{2}}
\end{array}\right]
$$


Electronic Journal of Linear Algebra, ISSN 1081-3810

with diagonal blocks

$$
B_{11}=\left[\begin{array}{cc}
0 & 0 \\
-\lambda_{n} & \lambda_{n}
\end{array}\right], \quad B_{k k}=\left[\begin{array}{cc}
0 & \lambda_{k} \\
-\lambda_{n-k+1} & \lambda_{k}+\lambda_{n-k+1}
\end{array}\right]
$$

and

$$
B_{k 1}=\left[\begin{array}{rr}
0 & -\lambda_{k} \\
0 & -\lambda_{k}
\end{array}\right], \quad k=2,3, \ldots, \frac{n}{2}
$$

Let $\mathbf{q}=\left(q_{1}, q_{2}, \ldots, q_{n}\right)^{T}$ with

$$
\begin{aligned}
q_{2 k-1} & =0, \quad k=1,2, \ldots, \frac{n}{2}, \\
q_{2} & =-\lambda_{n}, \\
q_{2 k} & =-\left(\lambda_{k}+\lambda_{n-k+1}\right), \quad k=2,3, \ldots, \frac{n}{2} .
\end{aligned}
$$

Then, $A=B+\mathbf{e q}^{\mathbf{T}}$ is nonnegative with spectrum $\Lambda$. The proof for odd $n$ is similar with the $1 \times 1$ blocks

$$
B_{\frac{n+1}{2} \frac{n+1}{2}}=\left[\lambda_{\frac{n+1}{2}}\right], \quad B_{\frac{n+1}{2} 2}=\left[-\lambda_{\frac{n+1}{2}}\right], \quad \text { where } \lambda_{\frac{n+1}{2}} \leq 0
$$

and $q_{n}=-\lambda_{\frac{n+1}{2}}$.

Theorem 4.3. (Ciarlet, [6]) Let $\Lambda=\left\{\lambda_{1}, \lambda_{2}, \ldots, \lambda_{n}\right\}$ such that

$$
\lambda_{1} \geq \lambda_{2} \geq \cdots \geq \lambda_{p} \geq 0>\lambda_{p+1} \geq \cdots \geq \lambda_{n} \quad \text { and } \quad 1 \leq p \leq n-1 .
$$

If $\left|\lambda_{k}\right| \leq \frac{\lambda_{1}}{n}, k=2,3, \ldots, n$, then $\Lambda$ is realizable.

Proof. Consider the matrix

$$
B=\left[\begin{array}{cccccccc}
0 & 0 & 0 & \cdots & 0 & 0 & \cdots & 0 \\
-\lambda_{2} & \lambda_{2} & 0 & \cdots & 0 & 0 & \cdots & 0 \\
-\lambda_{3} & 0 & \lambda_{3} & \cdots & 0 & 0 & \cdots & 0 \\
\vdots & \vdots & \vdots & \ddots & \vdots & \vdots & \ddots & \vdots \\
-\lambda_{p} & 0 & 0 & \cdots & \lambda_{p} & 0 & \cdots & 0 \\
-\lambda_{p+1} & 0 & 0 & \cdots & 0 & \lambda_{p+1} & \cdots & 0 \\
\vdots & \vdots & \vdots & \cdots & \vdots & \vdots & \ddots & \vdots \\
-\lambda_{n} & 0 & 0 & \cdots & 0 & 0 & \cdots & \lambda_{n}
\end{array}\right] \in \mathcal{C S}_{0}
$$

$B$ has spectrum $\left\{0, \lambda_{2}, \ldots, \lambda_{n}\right\}$. Then, for $\mathbf{q}=\left(\frac{\lambda_{1}}{n}, \ldots, \frac{\lambda_{1}}{n}\right)^{T}$, we have that $A=B+\mathbf{e q}^{\mathbf{T}}$ has spectrum $\Lambda$, and since $\left|\lambda_{k}\right| \leq \frac{\lambda_{1}}{n}, k=2,3, \ldots, n, A$ is nonnegative.

Theorem 4.4. (Kellogg, [18]) Let $\Lambda=\left\{\lambda_{1}, \lambda_{2}, \ldots, \lambda_{n}\right\}$ be a list of real numbers with $\lambda_{1} \geq \lambda_{2} \geq \cdots \geq \lambda_{n}$ and let $p$ be the largest index $j(1 \leq j \leq n)$ for which $\lambda_{j} \geq 0$. Let the set of indices

$$
K=\left\{i: \lambda_{i} \geq 0 \text { and } \lambda_{i}+\lambda_{n-i+2}<0, i \in\left\{2,3, \ldots,\left[\frac{n+1}{2}\right]\right\}\right\} .
$$




$$
\lambda_{1} \geq-\sum_{i \in K, \quad}\left(\lambda_{i}+\lambda_{n-i+2}\right)-\lambda_{n-k+2} \quad \text { for all } k \in K,
$$

and

$$
\lambda_{1} \geq-\sum_{i \in K}\left(\lambda_{i}+\lambda_{n-i+2}\right)-\sum_{j=p+1}^{n-p+1} \lambda_{j}, \text { provided that } n \geq 2 p,
$$

then $\Lambda$ is realizable.

Proof. Suppose conditions (4.6) and (4.7) are satisfied. Let $K=\left\{k_{1}, k_{2}, \ldots, k_{t}\right\}$ be the Kellogg set of indices. Consider the partition $\Lambda=\Lambda_{1} \cup \cup_{i=1}^{t} \Lambda_{k_{i}} \cup \Lambda_{\mathcal{R}}$, where

$$
\begin{aligned}
\Lambda_{1} & =\left\{\lambda_{1}, \lambda_{p+1}, \ldots, \lambda_{n-p+1}\right\}, \\
\Lambda_{k_{i}} & =\left\{\lambda_{k_{i}}, \lambda_{n-k_{i}+2}\right\}, \quad k_{i} \in K, \quad i=1,2, \ldots, t \quad\left(\lambda_{k_{1}} \geq \lambda_{k_{2}} \geq \cdots \geq \lambda_{k_{t}} \geq 0\right), \\
\Lambda_{\mathcal{R}} & =\Lambda-\Lambda_{1}-\cup_{i=1}^{t} \Lambda_{k_{i}} .
\end{aligned}
$$

From (4.7), we have that

$$
\lambda_{1}+\sum_{j=p+1}^{n-p+1} \lambda_{j} \geq-\sum_{i \in K}\left(\lambda_{i}+\lambda_{n-i+2}\right)>0 \quad\left(\left(\lambda_{i}+\lambda_{n-i+2}\right)<0 \quad \forall i \in K\right) .
$$

Since $\lambda_{p+1}, \lambda_{p+2}, \ldots, \lambda_{n-p+1}$ are negative, from Theorem $4.1, \Lambda_{1}$ is realizable.

If $\Lambda_{\mathcal{R}}=\emptyset$ then $\Lambda=\Lambda_{1} \cup \cup_{i=1}^{t} \Lambda_{k_{i}}$. If $\Lambda_{\mathcal{R}} \neq \emptyset$, then $\Lambda_{\mathcal{R}}$ contain sublists $\Lambda_{i}=\left\{\lambda_{i}, \lambda_{n-i+2}\right\}$ such that $\left(\lambda_{i}+\lambda_{n-i+2}\right) \geq 0$. So, the lists $\Lambda_{i}$ are realizable by

$$
A_{i}=\left[\begin{array}{cc}
0 & \lambda_{i} \\
-\lambda_{n-i+2} & \lambda_{i}+\lambda_{n-i+2}
\end{array}\right] .
$$

Let $A_{\mathcal{R}}$ be the realizing matrix of $\Lambda_{\mathcal{R}}$. On the other hand, for each sublist $\Lambda_{k_{i}}=\left\{\lambda_{k_{i}}, \lambda_{n-k_{i}+2}\right\}$ with $k_{i} \in K$, $i=1,2, \ldots, t$,

$$
B_{k_{i}}=\left[\begin{array}{cc}
0 & \lambda_{k_{i}} \\
-\lambda_{n-k_{i}+2} & \lambda_{k_{i}}+\lambda_{n-k_{i}+2}
\end{array}\right]
$$

is a $2 \times 2$ matrix with spectrum $\Lambda_{k_{i}}, i=1,2, \ldots, t$. Since $\lambda_{k_{i}} \geq 0$ and $\left(\lambda_{k_{i}}+\lambda_{n-k_{i}+2}\right)<0$, then

$$
-\lambda_{n-k_{i}+2}>0
$$

Let

$$
\Lambda_{1}^{\prime}=\left\{\lambda_{1}+\sum_{i=1}^{t}\left(\lambda_{k_{i}}+\lambda_{n-k_{i}+2}\right), \lambda_{p+1}, \ldots, \lambda_{n-p+1}\right\},
$$

From (4.7), $\Lambda_{1}^{\prime}$ is realizable by an $(n-2 p+2) \times(n-2 p+2)$ matrix $B_{1}^{\prime} \in \mathcal{C S}_{\mu}$, where

$$
\mu=\lambda_{1}+\sum_{i=1}^{t}\left(\lambda_{k_{i}}+\lambda_{n-k_{i}+2}\right) \text {. }
$$


Electronic Journal of Linear Algebra, ISSN 1081-3810

A publication of the International Linear Algebra Society

Volume 36, pp. 484-502, July 2020.

Now, let

$$
B=\left[\begin{array}{ccccccc}
B_{1}^{\prime} & 0 & 0 & \cdots & \cdots & 0 & 0 \\
B_{2} & B_{k_{t}} & 0 & \ddots & \vdots & \vdots & \vdots \\
0 & B_{k_{t-1}}(\mu) & B_{k_{t-1}} & \ddots & \ddots & \vdots & \vdots \\
\vdots & B_{k_{t-2}}(\mu) & B_{k_{t-1}}^{\prime} & B_{k_{t-2}} & \ddots & \ddots & \vdots \\
\vdots & \vdots & \vdots & \vdots & \ddots & 0 & 0 \\
\vdots & B_{k_{2}}(\mu) & B_{k_{t-1}}^{\prime} & B_{k_{t-2}}^{\prime} & \cdots & B_{k_{2}} & 0 \\
0 & B_{k_{1}}(\mu) & B_{k_{t-1}}^{\prime} & B_{k_{t-2}}^{\prime} & \cdots & B_{k_{2}}^{\prime} & B_{k_{1}}
\end{array}\right]
$$

where

$$
\begin{gathered}
B_{2}=\left[\begin{array}{llll}
0 & \cdots & 0 & \mu-\lambda_{k_{t}} \\
0 & \cdots & 0 & \mu-\lambda_{k_{t}}
\end{array}\right]_{2 \times(n-2 p+2)}, \\
B_{k_{i}}=\left[\begin{array}{cc}
0 & \lambda_{k_{i}} \\
-\lambda_{n-k_{i}+2} & \lambda_{k_{i}}+\lambda_{n-k_{i}+2}
\end{array}\right], \quad i=1,2, \ldots, t, \\
B_{k_{i}}(\mu)=\left[\begin{array}{c}
\mu-\lambda_{k_{i}}-\sum_{j=i+1}^{t-1}\left(\lambda_{k_{j}}+\lambda_{n-k_{j}+2}\right) \\
0 \quad \mu-\lambda_{k_{i}}-\sum_{j=i+1}^{t-1}\left(\lambda_{k_{j}}+\lambda_{n-k_{j}+2}\right)
\end{array}\right], \quad i=1,2, \ldots, t-1, \\
B_{k_{i}}^{\prime}=\left[\begin{array}{ll}
0 & \lambda_{k_{i}}+\lambda_{n-k_{i}+2} \\
0 & \lambda_{k_{i}}+\lambda_{n-k_{i}+2}
\end{array}\right], \quad i=2,3, \ldots, t-1,
\end{gathered}
$$

are such that $B \in \mathcal{C S}_{\mu}$ with spectrum $\Lambda_{1}^{\prime} \cup \cup_{i=1}^{t} \Lambda_{k_{i}}$.

Note that the sum $\sum_{j=i+1}^{t-1}\left(\lambda_{k_{j}}+\lambda_{n-k_{j}+2}\right)$ in the matrix $B_{k_{i}}(\mu)$ is zero if $i+1>t-1$ for some $i$. From Theorem 2.1, we have for

$$
\mathbf{q}=(\underbrace{0,0, \ldots, 0}_{(n-2 p+2)-\text { times }}, 0,-\left(\lambda_{k_{t}}+\lambda_{n-k_{t}+2}\right), \ldots, 0,-\left(\lambda_{k_{1}}+\lambda_{n-k_{1}+2}\right))^{T}
$$

that $M=B+\mathbf{e q}^{\mathbf{T}} \in \mathcal{C} \mathcal{S}_{\lambda_{1}}$ is nonnegative with spectrum $\Lambda_{1} \cup \cup_{i=1}^{t} \Lambda_{k_{i}}$.

Finally, a matrix $A=M \oplus A_{\mathcal{R}}$ is nonnegative with spectrum $\Lambda$.

Before proving Borobia's result from Theorem 2.1we need the following result, which was proved in [35]: Lemma 4.5. Let

$$
B_{k}=\left[\begin{array}{cc}
0 & \lambda_{k} \\
-\lambda_{n-k+2} & \lambda_{k}+\lambda_{n-k+2}
\end{array}\right]
$$


where $\lambda_{n-k+2}=\sum_{j=1}^{r} \mu_{j}<0$ with $\mu_{j}<0, j=1,2, \ldots, r$. Then

$$
\Lambda_{k}=\left\{\lambda_{k}, \lambda_{n-k+2}\right\} \quad \text { and } \quad \Lambda_{r}=\left\{\lambda_{k}, \mu_{1}, \ldots, \mu_{r}\right\}
$$

have the same Brauer's negativity, $\mathcal{N}\left(\Lambda_{k}\right)=\mathcal{N}\left(\Lambda_{r}\right)$. Moreover, there exists a matrix $B_{r} \in \mathcal{C} \mathcal{S}_{\lambda_{k}}$, of order $r+1$, with spectrum $\Lambda_{r}$.

TheOREM 4.6. (Borobia, [1]) Let $\Lambda=\left\{\lambda_{1}, \lambda_{2}, \ldots, \lambda_{n}\right\}$ be a list of real numbers with $\lambda_{1} \geq \lambda_{2} \geq \ldots \geq$ $\lambda_{p} \geq 0>\lambda_{p+1} \geq \cdots \geq \lambda_{n}$ (p is the largest index $j\left(1 \leq j \leq n\right.$ ) for which $\lambda_{j} \geq 0$. ) If there exists a partition $J_{1} \cup J_{2} \cup \cdots \cup J_{t}$ of $J=\left\{\lambda_{p+1}, \lambda_{p+2}, \ldots, \lambda_{n}\right\}$, for some $1 \leq t \leq n-p$, such that

$$
\lambda_{1} \geq \lambda_{2} \geq \cdots \geq \lambda_{p} \geq \sum_{\lambda \in J_{1}} \lambda \geq \sum_{\lambda \in J_{2}} \lambda \geq \cdots \geq \sum_{\lambda \in J_{t}} \lambda
$$

satisfies the Kellogg conditions (4.6) and (4.7), then $\Lambda$ is realizable.

Proof. Suppose that Borobia realizability criterion is satisfied. Then, there exists a partition

$$
J_{1} \cup J_{2} \cup \cdots \cup J_{t} \quad \text { of } J=\left\{\lambda_{p+1}, \ldots, \lambda_{n}\right\} \text {, }
$$

with

$$
\sum_{\lambda \in J_{j}} \lambda=\mu_{p+j}, \quad j=1,2, \ldots, t, \quad 1 \leq t \leq n-p
$$

such that the new list

$$
\Gamma=\left\{\mu_{1}, \mu_{2}, \ldots, \mu_{p}, \mu_{p+1}, \ldots, \mu_{p+t}\right\}
$$

with $\mu_{1} \geq \cdots \geq \mu_{p} \geq 0>\mu_{p+1} \geq \cdots \geq \mu_{p+t}$, where $\mu_{i}=\lambda_{i}, i=1, \ldots, p$, satisfies the Kellogg realizability criterion.

Now, we apply the same proof as in Theorem 4.4, except for one detail. Since the new list

$$
\Gamma=\left\{\mu_{1}, \ldots, \mu_{p}, \mu_{p+1}, \ldots, \mu_{p+t}\right\}
$$

has less elements than the original list $\Lambda=\left\{\lambda_{1}, \lambda_{2}, \ldots, \lambda_{n}\right\}$ and we want to obtain a nonnegative matrix of order $n$ realizing $\Lambda$, then, in each step of the proof, before manipulating any of the new sublists

$$
\Gamma_{k_{i}}=\left\{\mu_{k_{i}}, \mu_{p+t-k_{i}+2}\right\}, \quad k_{i} \in K \quad \text { (Kellogg set of indices) }
$$

where

$$
\mu_{p+t-k_{i}+2}=\mu_{p+j}=\sum_{\lambda \in J_{j}} \lambda<0, \quad \forall k_{i} \in K \quad \text { and } \quad j=1, \ldots, t
$$

we must extend $\Gamma_{k_{i}}$ to the lists $\Gamma_{r_{i}}=\left\{\mu_{k_{i}}, \lambda_{1_{j}}, \ldots, \lambda_{r_{j}}\right\}, \forall k_{i} \in K$, where

$$
\lambda_{s_{j}} \in J_{j}, \quad s=1, \ldots, r, \quad \text { and } \quad \sum_{s=1}^{r} \lambda_{s_{j}}=\mu_{p+t-k_{i}+2}, \quad j=1, \ldots, t .
$$


Electronic Journal of Linear Algebra, ISSN 1081-3810

A publication of the International Linear Algebra Society

Volume 36, pp. 484-502, July 2020.

From Lemma $4.5 \mathcal{N}\left(\Gamma_{k_{i}}\right)=\mathcal{N}\left(\Gamma_{r_{i}}\right)$. Then, the realizing matrix for $\Gamma_{r_{i}}$ is of the required size. In the same way, if necessary, we must also extend the new list

$$
\Gamma_{1}^{\prime}=\left\{\mu, \mu_{p+1}, \ldots, \mu_{t+1}\right\}, \quad \text { with } \mu=\mu_{1}+\sum_{k_{i} \in K}\left(\mu_{k_{i}}+\mu_{p+t-k_{i}+2}\right)
$$

to a new list $\Gamma_{1}^{\prime \prime}$ with the same Brauer's negativity, by replacing the corresponding $\mu_{p+1}, \ldots, \mu_{t+1}$ by $\sum_{\lambda \in J_{1}} \lambda, \ldots, \sum_{\lambda \in J_{t-p+1}} \lambda$, respectively. Again, from Lemma 4.5, $\mathcal{N}\left(\Gamma_{1}^{\prime}\right)=\mathcal{N}\left(\Gamma_{1}^{\prime \prime}\right)$. Thus, the realizing matrix of $\Gamma_{1}^{\prime \prime}$ has the required size. Finally, from Suleimanova criterion, is easy to construct the realizing matrices for $\Gamma_{r_{i}}, \Gamma_{1}^{\prime \prime}$ and $\Gamma_{\mathcal{R}}$, and, analogously as in proof of Theorem 4.4, we use Theorem 2.1for constructing a nonnegative matrix with spectrum $\Lambda$.

REMARK 4.7. Some realizability criteria, such as those of Kellogg and Borobia, give sufficient conditions for the existence of a solution, but not for its construction, as with the criteria from Theorem 2.1 and Theorem 2.4

The following result, by Šmigoc [33], can be proved by using Theorem 2.4.

ThEOREM 4.8. ([33, Theorem 10]) Let $A=\left[\begin{array}{ll}A_{1} & \mathbf{a} \\ \mathbf{b}^{T} & c\end{array}\right]$ be an $n \times n$ nonnegative matrix with spectrum $\Lambda$ and let $B$ be an $m \times m$ nonnegative matrix with Perron eigenvalue $\lambda_{1}$, spectrum $\left\{\lambda_{1}\right\} \cup \Lambda^{\prime}$ and maximal diagonal element $d$. If $\lambda_{1} \leq c$, then there exists an $(n+m-1) \times(n+m-1)$ nonnegative matrix $M$ with spectrum $\Lambda \cup \Lambda^{\prime}$ and maximal diagonal element greater than or equal to $c+d-\lambda_{1}$.

Proof. We suppose, without loss of generality, that $B \in \mathcal{C S}_{\lambda_{1}}$ with maximal diagonal element $d$ in the position $b_{m m}$. If $\lambda_{1} \leq c$, we take $\epsilon=c-\lambda_{1} \geq 0$, and from Theorem 2.1 there exists a nonnegative matrix $B^{\prime}=B+\epsilon \mathbf{e e}_{n}$ with spectrum $\left\{c, \Lambda^{\prime}\right\}$ and maximal diagonal element $d+c-\lambda_{1}$. On the other hand, let $A$ be a nonnegative matrix with spectrum $\Lambda$ and diagonal entries $a_{2}, a_{3}, \ldots, a_{n}, c$. From Theorem 2.4 , the $(n+m-1) \times(n+m-1)$ matrix

$$
M=\left[\begin{array}{lllllllll}
a_{2} & & & & \\
& a_{3} & & & \\
& & \ddots & & \\
& & & a_{n} & \\
& & & B^{\prime}
\end{array}\right]+X C, \text { with } X=\left[\begin{array}{ccccc}
1 & 0 & \cdots & 0 & 0 \\
0 & 1 & \cdots & 0 & 0 \\
\vdots & 0 & \ddots & \vdots & \vdots \\
\vdots & \vdots & \ddots & 1 & 0 \\
\vdots & \vdots & \cdots & 0 & 1 \\
\vdots & \vdots & \cdots & 0 & \vdots \\
\vdots & \vdots & \cdots & \vdots & \vdots \\
0 & \cdots & \cdots & 0 & 1
\end{array}\right]_{(n+m-1) \times n}
$$

has spectrum $\left\{\Lambda, \Lambda^{\prime}\right\}$, where $\Lambda$ is the spectrum of $\Omega+C X=A$, being $\Omega=\operatorname{diag}\left\{a_{2}, a_{3}, \ldots, a_{n}, c\right\}$. Since $C X=A-\Omega \geq 0$, it is clear that the $n \times(n+m-1)$ matrix

$$
C=\left[\begin{array}{ccc}
A_{1}^{\prime} & \mathbf{a} & 0 \cdots 0 \\
\mathbf{b}^{T} & 0 & 0 \cdots 0
\end{array}\right] \quad\left(A_{1}^{\prime} \text { is } A_{1} \text { with } \operatorname{tr}\left(A_{1}\right)=0\right)
$$

is nonnegative. Therefore, the matrix $M$ is nonnegative. Finally, from the construction of $M$, it is clear that $M$ has a maximal diagonal element greater than or equal to $c+d-\lambda_{1}$. 
REMARK 4.9. In [22], the authors construct a map of sufficient conditions for the RNIEP to have a solution, with inclusion relations or independency relations between them. There, they point out that Soto 2 , Perfect $2^{+}$, and Soto-Rojo realizability criteria (all them obtained from results by Brauer and Rado), are the most general criteria. In particular, they conclude that Soto-Rojo criterion, by its own nature, trivially contains all realizability criteria, which are compared in [22].

5. SNIEP. In this section, we consider the symmetric nonnegative inverse eigenvalue problem $(S N I E P)$. It is well known that the RNIEP and the SNIEP are equivalent for $n \leq 4$, while they are different for $n \geq 5$ [13]. The first results about symmetric nonnegative realizations are due to Fiedler [10]. Several realizability criteria obtained for the NIEP, have later been proved to be also symmetric realizability criteria. Fiedler and Radwan, in [10] and [29], respectively, show that Kellogg and Borobia realizability criteria are also symmetric realizability criteria. In [36, 38], it is shown that the NIEP realizability criteria given in [34], are also symmetric realizability criteria. In [39], the authors prove Theorem 2.6, which gives a symmetric version of Theorem 2.4. Then, by applying Theorem 2.6, they prove a new symmetric realizability criterion [39, Theorems 2.6 and 3.1], which strictly contains criteria in [34]. In [43], it is also shown that the criteria called Family Soto p criteria are also NIEP and SNIEP realizability criteria (see $[9,23])$.

Next, by the use of Theorem 2.6, we give alternative proofs for the following two results of Fiedler:

Lemma 5.1. (Fiedler, [10]) Let $A$ be a symmetric $m \times m$ matrix with spectrum $\Lambda_{1}=\left\{\alpha_{1}, \ldots, \alpha_{m}\right\}$. Let $\mathbf{u}=\left(u_{1}, \ldots, u_{m}\right),\|\mathbf{u}\|=1$, be a unit eigenvector of $A$ corresponding to $\alpha_{1}$. Let $B$ be a symmetric $n \times n$ matrix with spectrum $\Lambda_{2}=\left\{\beta_{1}, \ldots, \beta_{n}\right\}$. Let $\mathbf{v}=\left(v_{1}, \ldots, v_{n}\right),\|\mathbf{v}\|=1$, be a unit eigenvector of $B$ corresponding to $\beta_{1}$. Then for any scalar $\rho$, the matrix

$$
C=\left[\begin{array}{cc}
A & \rho \mathbf{u v} \\
\rho \mathbf{v} \mathbf{u}^{\mathbf{T}} & B
\end{array}\right]
$$

has spectrum $\Lambda=\left\{\gamma_{1}, \gamma_{2}, \alpha_{2}, \ldots, \alpha_{m}, \beta_{2}, \ldots, \beta_{n}\right\}$, where $\gamma_{1}, \gamma_{2}$ are eigenvalues of the matrix

$$
\widehat{C}=\left[\begin{array}{cc}
\alpha_{1} & \rho \\
\rho & \beta_{1}
\end{array}\right]
$$

Proof. The matrix $M=\left[\begin{array}{cc}A & 0 \\ 0 & B\end{array}\right]$ is symmetric of order $(m+n)$ with eigenvalues $\alpha_{1}, \ldots, \alpha_{m}, \beta_{1}, \ldots, \beta_{n}$. Let

$$
X_{1}^{T}=\left(u_{1}, \ldots, u_{m}, 0, \ldots, 0\right) \quad \text { and } \quad X_{2}^{T}=\left(0, \ldots, 0, v_{1}, \ldots, v_{n}\right)
$$

$(m+n)$-dimensional vectors. Let $X=\left[X_{1} \mid X_{2}\right]$ and $\Omega=\operatorname{diag}\left\{\alpha_{1}, \beta_{1}\right\}$. Then $M X=X \Omega$. Let

$$
C=\left[\begin{array}{ll}
0 & \rho \\
\rho & 0
\end{array}\right] .
$$

Then

$$
X C X^{T}=\left[\begin{array}{cc}
0 & \rho \mathbf{u v}^{T} \\
\rho \mathbf{v} \mathbf{u}^{T} & 0
\end{array}\right]
$$


and the matrix

$$
M+X C X^{T}=\left[\begin{array}{cc}
A & \rho \mathbf{u v} \mathbf{v}^{T} \\
\rho \mathbf{v} \mathbf{u}^{T} & B
\end{array}\right]
$$

is symmetric. From Theorem 2.6, it has spectrum

$$
\Lambda=\left\{\gamma_{1}, \gamma_{2}, \alpha_{2}, \ldots, \alpha_{m}, \beta_{2}, \ldots, \beta_{n}\right\},
$$

where $\gamma_{1}$ and $\gamma_{2}$ are eigenvalues of the matrix

$$
\Omega+C=\left[\begin{array}{cc}
\alpha_{1} & \rho \\
\rho & \beta_{1}
\end{array}\right]
$$

for any $\rho$.

Observe that Theorem 2.6 generalizes Lemma 5.1 by Fiedler. In fact, if we have symmetric matrices $A_{1}, A_{2}, \ldots, A_{p}$, with corresponding spectra $\Lambda_{i}=\left\{\alpha_{1}^{(i)}, \alpha_{2}^{(i)}, \ldots, \alpha_{n_{i}}^{(i)}\right\}, i=1,2, \ldots, p$, and unitary eigenvectors $\mathbf{u}^{(i)}$ associated, respectively, to the eigenvalues $\alpha_{1}^{(i)}$, then from Theorem 2.6, we may obtain a symmetric $n \times n$ matrix

$$
A=\left(A_{1} \oplus A_{2} \oplus \cdots \oplus A_{p}\right)+X C X^{T},
$$

with spectrum $\left\{\gamma_{1}, \ldots, \gamma_{p}, \alpha_{2}^{(1)}, \ldots, \alpha_{n_{1}}^{(1)}, \ldots, \alpha_{2}^{(p)}, \ldots, \alpha_{n_{p}}^{(p)}\right\}$, where $\gamma_{1}, \ldots, \gamma_{p}$ are eigenvalues of the matrix $\Omega+C$, with $\Omega=\operatorname{diag}\left\{\alpha_{1}^{(1)}, \alpha_{1}^{(2)}, \ldots, \alpha_{1}^{(p)}\right\}$.

In what follows $\mathcal{S}_{n}\left(\widehat{\mathcal{S}_{n}}\right)$ denote the set of all lists $\Lambda$ for which there exists an $n \times n$ symmetric nonnegative (positive) matrix with spectrum $\Lambda$.

Theorem 5.2. (Fiedler, [10]) If

$$
\Lambda_{1}=\left\{\alpha_{1}, \ldots, \alpha_{m}\right\} \in \mathcal{S}_{m}, \quad \Lambda_{2}=\left\{\beta_{1}, \ldots, \beta_{n}\right\} \in \mathcal{S}_{n}
$$

and $\alpha_{1} \geq \beta_{1}$, then for any $\epsilon \geq 0$,

$$
\Lambda=\left\{\alpha_{1}+\epsilon, \beta_{1}-\epsilon, \alpha_{2}, \ldots, \alpha_{m}, \beta_{2}, \ldots, \beta_{n}\right\} \in \mathcal{S}_{m+n} .
$$

Proof. If $\Lambda_{1} \in \mathcal{S}_{m}$ and $\Lambda_{2} \in \mathcal{S}_{n}$, then there exist symmetric nonnegative matrices $A$ and $B$, with spectrum $\Lambda_{1}$ and $\Lambda_{2}, A \mathbf{u}=\alpha_{1} \mathbf{u}, B \mathbf{v}=\beta_{1} \mathbf{v},\|\mathbf{u}\|=\|\mathbf{v}\|=1$, respectively. From Theorem 2.6 we have

$$
M=\left[\begin{array}{cc}
A & 0 \\
0 & B
\end{array}\right], \quad \text { with } M X=X \Omega
$$

where

$$
\Omega=\left[\begin{array}{cc}
\alpha_{1} & 0 \\
0 & \beta_{1}
\end{array}\right] \text { and } X=\left[\begin{array}{ll}
\mathbf{u} & 0 \\
0 & \mathbf{v}
\end{array}\right]
$$

Moreover, for

$$
C=\left[\begin{array}{ll}
0 & \rho \\
\rho & 0
\end{array}\right], \quad \rho>0
$$




$$
M+X C X^{T}=\left[\begin{array}{cc}
A & \rho \mathbf{u v} \mathbf{v}^{\mathbf{T}} \\
\rho \mathbf{v} \mathbf{u}^{\mathbf{T}} & B
\end{array}\right]
$$

is symmetric nonnegative with spectrum $\left\{\gamma_{1}, \gamma_{2}, \alpha_{2}, \ldots, \alpha_{m}, \beta_{2}, \ldots, \beta_{n}\right\}$, where $\gamma_{1}$ and $\gamma_{2}$ are eigenvalues of $\Omega+C=\left[\begin{array}{cc}\alpha_{1} & \rho \\ \rho & \beta_{1}\end{array}\right]$. If we choose $\rho=\sqrt{\epsilon\left(\epsilon+\left(\alpha_{1}-\beta_{1}\right)\right)}$, then, $\gamma_{1}$ and $\gamma_{2}$ are obtained as $\alpha_{1}+\epsilon$ and $\beta_{1}-\epsilon$, respectively.

It is known that if $\Lambda=\left\{\lambda_{1}, \lambda_{2}, \ldots, \lambda_{n}\right\} \in \mathcal{S}_{n}$ and $\epsilon>0$, then $\Lambda_{\epsilon}=\left\{\lambda_{1}+\epsilon, \lambda_{2}, \ldots, \lambda_{n}\right\} \in \widehat{\mathcal{S}_{n}}$. It is clear that this result can be also proved by using Theorem 2.1.

REMARK 5.3. The criterion obtained from Theorem 2.6 is good for SNIEP (in general Theorem 2.1 destroy the symmetry of a matrix). In [23] the authors construct a map of sufficient conditions for SNIEP. Again, as for the RNIEP, the most general sufficient conditions for the SNIEP to have a solution, have been obtained from results by Brauer or Rado. In particular, the criterion given in [39], by its own nature, trivially contains any other realizability criterion for the SNIEP.

6. Complex NIEP. In this section, we consider a list $\Lambda=\left\{\lambda_{1}, \lambda_{2}, \ldots, \lambda_{n}\right\}$ of complex numbers. In [2] the authors introduce the following complex generalization of Theorem 4.1 by Suleimanova. The proof uses Theorem 2.1:

Theorem 6.1. ([2]) Let $\Lambda=\left\{\lambda_{1}, \lambda_{2}, \ldots, \lambda_{n}\right\}$, with $\Lambda=\bar{\Lambda}$ and

$$
\Lambda^{\prime}=\left\{\lambda_{2}, \ldots, \lambda_{n}\right\} \subset\{z \in \mathbb{C}: \operatorname{Re} z \leq 0,|\operatorname{Re} z| \geq|\operatorname{Im} z|\}
$$

Then, $\Lambda$ is realizable if and only if $\sum_{i=1}^{n} \lambda_{i} \geq 0$.

In [33], Šmigoc proved that (6.9) can be improved to

$$
\Lambda^{\prime}=\left\{\lambda_{2}, \ldots, \lambda_{n}\right\} \subset\{z \in \mathbb{C}: \operatorname{Re} z \leq 0, \sqrt{3}|\operatorname{Re} z| \geq|\operatorname{Im} z|\}
$$

Then, $\Lambda=\left\{\lambda_{1}, \lambda_{2}, \ldots, \lambda_{n}\right\}$ is also realizable if and only if $\sum_{i=1}^{n} \lambda_{i} \geq 0$.

Next, we give an alternative proof of a result by Šmigoc [33]. First, we need the following lemma, given in [41, Theorem 2.2].

LEMmA 6.2. The numbers $\omega_{1}, \omega_{2}, \omega_{3}$ and $\lambda_{1}, \lambda_{2}, \lambda_{3}\left(\lambda_{1} \geq\left|\lambda_{i}\right|, i=2,3\right)$ are, respectively, the diagonal entries and eigenvalues of a nonnegative matrix $B \in \mathcal{C S}_{\lambda_{1}}$ if only if

i) $0 \leq \omega_{k} \leq \lambda_{1}, k=1,2,3$,

ii) $\omega_{1}+\omega_{2}+\omega_{3}=\lambda_{1}+\lambda_{2}+\lambda_{3}$,

iii) $\omega_{1} \omega_{2}+\omega_{1} \omega_{3}+\omega_{2} \omega_{3} \geq \lambda_{1} \lambda_{2}+\lambda_{1} \lambda_{3}+\lambda_{2} \lambda_{3}$, and

iv) $\max \omega_{k} \geq \operatorname{Re} \lambda_{2}$.

Theorem 6.3. Let $\Lambda=\left\{\lambda_{1}, \lambda_{2}, \ldots, \lambda_{n}\right\}, \Lambda=\bar{\Lambda}, \lambda_{j}=a_{j}+i b_{j}$ with $a_{j} \leq 0, j=2,3, \ldots, n$, satisfying $\left|b_{j}\right| \leq-\sqrt{3} a_{j}$. Then, $\Lambda$ is realizable if only if $\sum_{k=1}^{n} \lambda_{k} \geq 0$.

Proof. The condition is necessary. Now, suppose that $\sum_{k=1}^{n} \lambda_{k} \geq 0$. We use induction on $\mathrm{n}$, with $n \geq 2$. 
Electronic Journal of Linear Algebra, ISSN 1081-3810

A publication of the International Linear Algebra Society

Volume 36, pp. 484-502, July 2020.

For $n=2, \Lambda=\left\{\lambda_{1}, \lambda_{2}\right\}$ must be a real list with $\lambda_{2}<0$. Then,

$$
A=\frac{1}{2}\left[\begin{array}{ll}
\lambda_{1}+\lambda_{2} & \lambda_{1}-\lambda_{2} \\
\lambda_{1}-\lambda_{2} & \lambda_{1}+\lambda_{2}
\end{array}\right]
$$

is nonnegative with spectrum $\Lambda$. For $n=3$ and $\Lambda=\left\{\lambda_{1}, \lambda_{2}, \lambda_{3}\right\}$ with $\lambda_{j}<0, j=2,3$, the conditions from Lemma 6.2 are satisfied and, therefore, there exists a nonnegative matrix with prescribed eigenvalues and diagonal entries.

If $\Lambda=\left\{\lambda_{1}, a+i b, a-i b\right\}$ with $a<0,|b| \leq-\sqrt{3} a, \lambda_{1}+2 a \geq 0$, then, since

$$
2 a \lambda_{1}+4 a^{2} \leq 0 \quad \text { and } \quad-3 a^{2}+b^{2} \leq 0
$$

we have $2 \lambda_{1} a+a^{2}+b^{2} \leq 0$. So, the conditions from Lemma 6.2 are also satisfied and, therefore, there exists a $3 \times 3$ nonnegative matrix with spectrum $\Lambda$ and the prescribed diagonal entries. Now, we suppose that $\Lambda$, with $m-2$ numbers, $4 \leq m \leq n$, is realizable. Let

$$
\Lambda^{\prime}=\left\{\lambda_{1}, \lambda_{2}, \ldots, \lambda_{m}\right\}, \text { with } \operatorname{Re} \lambda_{j} \leq 0, \quad \text { and } \quad \sqrt{3}\left|\operatorname{Re} \lambda_{j}\right| \geq\left|\operatorname{Im} \lambda_{j}\right|, \quad j=2, \ldots, m
$$

We take the partition

$$
\Lambda_{0}=\left\{\lambda_{1}, \lambda_{i}, \lambda_{j}\right\}, \quad \Lambda_{2}=\Lambda^{\prime}-\Lambda_{0}, \quad \Lambda_{1}=\Lambda_{3}=\emptyset
$$

with

$$
\Gamma_{2}=\left\{\lambda_{1}+\lambda_{i}+\lambda_{j}\right\} \cup \Lambda_{2}, \quad \Gamma_{1}=\Gamma_{3}=\{0\}
$$

where $\lambda_{i}, \lambda_{j}$ are real numbers or complex conjugated numbers. From the hypothesis of induction, $\Gamma_{2}$ is realizable by a nonnegative matrix $A_{2}$. Then

$$
A=\left[\begin{array}{lll}
A_{2} & & \\
& 0 & \\
& & 0
\end{array}\right]
$$

is nonnegative with spectrum $\Gamma_{2} \cup\{0,0\}$. From Lemma 6.2, we can construct a $3 \times 3$ nonnegative matrix $B$ with spectrum $\Lambda_{0}$ and diagonal entries $\left\{\lambda_{1}+\lambda_{i}+\lambda_{j}, 0,0\right\}$. Finally, from Theorem 2.4 with

$$
X=\left[\begin{array}{ccc}
\mathbf{x}_{2} & 0 & 0 \\
0 & 1 & 0 \\
0 & 0 & 1
\end{array}\right]_{m \times 3} \quad \text { and } \quad A_{2} \mathbf{x}_{\mathbf{2}}=\left(\lambda_{1}+\lambda_{i}+\lambda_{j}\right) \mathbf{x}_{\mathbf{2}}
$$

we have $B=\Omega+C X, \Omega=\operatorname{diag}\left\{\lambda_{1}+\lambda_{i}+\lambda_{j}, 0,0\right\}$. Then the $m \times m$ matrix

$$
M=\left[\begin{array}{lll}
A_{2} & & \\
& 0 & \\
& & 0
\end{array}\right]+X C
$$

has the spectrum $\Lambda=\left\{\lambda_{1}, \ldots, \lambda_{m}\right\}$. Moreover, since, $A, X$ and $C$ are nonnegative, $M$ is nonnegative. 
7. Universal realizability. We say that a list $\Lambda=\left\{\lambda_{1}, \ldots, \lambda_{n}\right\}$ of complex numbers is universally realizable, if it is realizable for each possible Jordan canonical form allowed by $\Lambda$. As far as we know, the first works on the universal realizability problem are due to Minc [25, 26]. In [26], Minc proves that if a list $\Lambda=\left\{\lambda_{1}, \ldots, \lambda_{n}\right\}$ of complex numbers has a diagonalizable positive realization, then $\Lambda$ is $U R$. Next, we give an alternative proof for this result.

Theorem 7.1. (Minc, [26]) Let $\Lambda=\left\{\lambda_{1}, \lambda_{2}, \ldots, \lambda_{n}\right\}$ be realizable by a diagonalizable positive matrix A. Then $\Lambda$ is universally realizable.

Proof. Let $A$ be positive with spectrum $\Lambda$ and let $S$ be a nonsingular matrix, such that $S^{-1} A S=J(A)$ is the diagonal Jordan canonical form of $A$. We perturb the diagonal matrix $J(A)$ by using Theorem 2.4. It is clear that the eigenvectors of $J(A)$ are the canonical vectors $\mathbf{e}_{1}, \mathbf{e}_{2}, \ldots, \mathbf{e}_{n}$. Let $\Omega=\operatorname{diag}\left\{\lambda_{2}, \lambda_{3}, \ldots, \lambda_{r+1}\right\}$ and consider the $n \times r$ matrix $X=\left[\mathbf{e}_{2}\left|\mathbf{e}_{3}\right| \cdots \mid \mathbf{e}_{r+1}\right]$ and the $r \times n$ matrix $C$ such that $\Omega+C X$ has eigenvalues $\lambda_{2}, \lambda_{3}, \ldots, \lambda_{r+1}$. Then $X C$ is of the form $\sum_{i \in K} E_{i, i+1}, K=\{2,3, \ldots, n-1\}$, and

$$
J(A)+X C=S^{-1} A S+X C=S^{-1}\left(A+S X C S^{-1}\right) S .
$$

By a convenient ordering of the columns, Minc [26] proved that the matrix $S X C S^{-1}$ is real. Hence, for $\epsilon>0$ small enough,

$$
M=A+\epsilon S X C S^{-1}
$$

is positive, with Jordan canonical form $J(M)=J(A)+X C$.

Results on the universal realizability problem, all of which have been obtained by applying Theorem 2.1 or Theorem 2.4, can be found in $[4,5,7,8,15,16,17,40,44]$. Other results give sufficient conditions for the universal realizability problem for structured matrices [45, 46, 47].

\section{REFERENCES}

[1] A. Borobia. On the Nonnegative Eigenvalue Problem. Linear Algebra Appl., 223/224:131-140, 1993.

[2] A. Borobia, J. Moro, and R.L. Soto. Negativity compensation in the nonnegative inverse eigenvalue problem. Linear Algebra Appl., 393:73-89, 2004.

[3] A. Brauer. Limits for the characteristic roots of a matrix. IV: Aplications to stochastic matrices. Duke Math. J., 19:75-91, 1952.

[4] J. Ccapa and R.L. Soto. On spectra perturbation and elementary divisors of positive matrices. Electron. J. Linear Algebra, 18:462-481, 2009.

[5] J. Ccapa nad R.L. Soto. On elementary divisors perturbation of nonnegative matrices. Linear Algebra Appl., 432:546-555, 2010.

[6] P.G. Ciarlet. Some results in the theory of nonnegative matrices. Linear Algebra Appl., 9:119-142, 1974.

[7] M. Collao, C.R. Johnson, and R.L. Soto. Universal realizability of spectra with two positive eigenvalues. Linear Algebra Appl., 545:226-239, 2018.

[8] R.C. Díaz and R.L. Soto. Nonnegative inverse elementary divisors problem in the left half plane. Linear Multilinear Algebra, 64:258-268, 2016.

[9] R. Ellard and H. Šmigoc. Connecting sufficient conditions for the symmetric nonnegative inverse eigenvalue problem. Linear Algebra Appl., 498:521-552, 2016.

[10] M. Fiedler. Eigenvalues of nonnegative symmetric matrices, Linear Algebra Appl., 9:119-142, 1974.

[11] W. Guo. Eigenvalues of nonnegative matrices. Linear Algebra Appl., 266:261-270, 1997.

[12] C.R. Johnson. Row stochastic matrices similar to doubly stochastic matrices. Linear Multilinear Algebra, 10:113-130, 1981.

[13] C.R. Johnson, T.J. Laffey, and R. Loewy. The real and the symmetric nonnegative inverse eigenvalue problems are different. Proc. Amer. Math. Soc., 124:3647-3651, 1996. 
[14] C.R. Johnson, C. Marijuán, and M. Pisonero. Ruling out certain 5-spectra for the symmetric nonnegative inverse eigenvalue problem. Linear Algebra Appl., 512:129-135, 2017.

[15] C.R. Johnson, A.I. Julio, and R.L. Soto. Nonnegative realizability with Jordan structure. Linear Algebra Appl., 587:302$313,2020$.

[16] A.I. Julio, C. Marijuán, M. Pisonero, and R.L. Soto. On universal realizability of spectra. Linear Algebra Appl., 563:353372,2019

[17] A.I. Julio and R.L. Soto. On the universal realizability problem. Linear Algebra Appl., 597:170-186, 2020.

[18] R. Kellogg. Matrices similar to a positive or essentially positive matrix. Linear Algebra Appl., 4:191-204, 1971.

[19] T.J. Laffey and E. Meehan. A characterization of trace zero nonnegative $5 \times 5$ matrices. Linear Algebra Appl., 302/303:295302, 1999.

[20] R. Loewy and D. London. A note on an inverse problem for nonnegative matrices. Linear Multilinear Algebra, 6:83-90, 1978.

[21] R. Loewy and O. Spector. Some notes on the spectra of nonnegative symmetric $5 \times 5$ matrices. Linear Multilinear Algebra, DOI: 10.1080/03081087.2019.1646205, 2019.

[22] C. Marijuán, M. Pisonero, and R.L. Soto. A map of sufficient conditions for the real nonnegative inverse eigenvalue problem. Linear Algebra Appl., 426:690-705, 2007.

[23] C. Marijuán, M. Pisonero, and R.L. Soto. A map of sufficient conditions for the symmetric nonnegative inverse eigenvalue problem. Linear Algebra Appl., 530:344-365, 2017.

[24] M.E. Meehan. Some Results on Matrix Spectra. Ph.D. Thesis, National University of Ireland, Dublin, 1998.

[25] H. Minc. Inverse elementary divisors problem for doubly stochastic matrices. Proc. Amer. Math. Soc., 83:665-670, 1981.

[26] H. Minc. Inverse elementary divisors problem for nonnegative matrices. Linear Multilinear Algebra, 11:121-131, 1982.

[27] H. Perfect. Methods of constructing certain stochastic matrices. Duke Math. J., 20:395-404, 1953.

[28] H. Perfect. Methods of constructing certain stochastic matrices II. Duke Math. J., 22:305-311, 1955.

[29] N. Radwan. An inverse eigenvalue problem for symmetric and normal matrices. Linear Algebra Appl., 248:101-109, 1996.

[30] R. Reams. An inequality for nonnegative matrices and the inverse eigenvalue problem. Linear Multilinear Algebra, 41:367375, 1996.

[31] O. Rojo and R.L. Soto. Existence and construction of nonnegative matrices with complex spectrum. Linear Algebra Appl., 368:53-69, 2003.

[32] F. Salzmann. A note on eigenvalues of nonnegative matrices. Linear Algebra Appl., 5:329-338, 1972.

[33] H. Šmigoc. The inverse eigenvalue problem for nonnegative matrices. Linear Algebra Appl., 393:365-374, 2004.

[34] R. Soto. Existence and construction of nonnegative matrices with prescribed spectrum. Linear Algebra Appl., 369:169-184, 2003.

[35] R.L. Soto, A. Borobia, and J. Moro. On the comparison of some realizability criteria for the real nonnegative inverse eigenvalue problem. Linear Algebra Appl., 396:223-241, 2005.

[36] R.L. Soto. Realizability by symmetric nonnegative matrices, Proyecciones Journal of Mathematics, 24:65-78, 2005.

[37] R.L. Soto and O. Rojo. Applications of a Brauer Theorem in the nonnegative inverse eigenvalue problem. Linear Algebra Appl., 416:844-856, 2006.

[38] R.L. Soto. Realizability criterion for the symmetric nonnegative inverse eigenvalue problem. Linear Algebra Appl., 416:783794, 2006.

[39] R.L. Soto, O. Rojo, J. Moro, and A. Borobia. Symmetric nonnegative realization of spectra. Electron. J. Linear Algebra, 16:1-18, 2007.

[40] R.L. Soto and J. Ccapa. Nonnegative matrices with prescribed elementary divisors. Electron. J. Linear Algebra, 17:287303, 2008.

[41] R.L. Soto, M. Salas, and C. Manzaneda. Nonnegative realization of complex spectra. Electron. J. Linear Algebra, 20:595609, 2010.

[42] R.L. Soto, O. Rojo, and C.B. Manzaneda. On nonnegative realization of partitioned spectra. Electron. J. Linear Algebra, 22:557-572, 2011.

[43] R.L. Soto. A family of realizability criteria for the real and symmetric nonnegative inverse eigenvalue problem. Nunerical Linear Algebra with Applications, 20:336-348, 2013.

[44] R.L. Soto, R.C. Díaz, H. Nina, and M. Salas. Nonnegative matrices with precribed spectrum and elementary divisors. Linear Algebra Appl., 439:3591-3604, 2013.

[45] R.L. Soto, A.I. Julio, and M. Salas. Nonnegative persymmetric matrices with prescribed elementary divisors. Linear Algebra Appl., 483:139-157, 2015.

[46] R.L. Soto, E. Valero, M. Salas, and H. Nina. Nonnegative generalized doubly stochastic matrices with prescribed elementary divisors. Electron. J. Linear Algebra, 30:704-720, 2015.

[47] R.L. Soto, R.C. Díaz, M. Salas, and O. Rojo. M-matrices with prescribed elementary divisors. Inverse Problems, 33:095009, 2017. 
Electronic Journal of Linear Algebra, ISSN 1081-3810

A publication of the International Linear Algebra Society

Volume 36, pp. 484-502, July 2020 .

[48] O. Spector. A characterization of trace zero symmetric nonnegative $5 \times 5$ matrices. Linear Algebra Appl., 434:1000-1017, 2011.

[49] H.R. Suleimanova. Stochastic matrices with real characteristic values. Dokl. Akad. Nauk SSSR, 66:343-345, 1949.

[50] J. Torre-Mayo, M.R. Abril-Raymundo, E. Alarcia-Estévez, C. Marijuán, and M. Pisonero. The nonnegative inverse eigenvalue problem from the coeffcients of the characteristic polynomial. EBL diagraphs. Linear Algebra Appl., 426:729-773, 2007. 